\title{
Network Meta-analysis of Food and Drug Administration-approved Treatment Options for Adults with Aquaporin-4 Immunoglobulin G-positive Neuromyelitis Optica Spectrum Disorder
}

\author{
Dean M. Wingerchuk · Ina Zhang · Adrian Kielhorn · Minying Royston • \\ Michael Levy · Kazuo Fujihara · Ichiro Nakashima · Imran Tanvir • \\ Friedemann Paul $\cdot$ Sean J. Pittock (D)
}

Received: August 11, 2021 / Accepted: October 18, 2021 / Published online: November 13, 2021

(C) The Author(s) 2021, corrected publication 2022

\begin{abstract}
Introduction: Neuromyelitis optica spectrum disorder (NMOSD) is an autoimmune disease defined by attacks on the central nervous system that cause irreversible damage. Recent approval of NMOSD therapies warrants investigations of comparative efficacy to inform treatment decisions.

Methods: A network meta-analysis (NMA) of all U.S. Food and Drug Administration-approved therapies (eculizumab, inebilizumab, and satralizumab) for adults with aquaporin-4 immunoglobulin G-positive (AQP4+) NMOSD was conducted via a systematic literature review
\end{abstract}

Supplementary Information The online version contains supplementary material available at https:// doi.org/10.1007/s40120-021-00295-8.

D. M. Wingerchuk

Mayo Clinic, Scottsdale, AZ, USA

I. Zhang

PRECISIONheor, Oakland, CA, USA

A. Kielhorn · M. Royston · I. Tanvir

Alexion, AstraZeneca Rare Disease, Boston, MA, USA

M. Levy

Massachusetts General Hospital, Boston, MA, USA

K. Fujihara

Fukushima Medical University, Fukushima City, Japan
(SLR) using data from randomized controlled trials (RCTs). Database searches of MEDLINE, EMBASE, and the Cochrane Central Register of Controlled Trials were executed for the SLR. A fixed-effects proportional hazards Bayesian NMA was used to estimate relative treatment effects based on data extracted from RCTs identified during the SLR (search end date: 11 September 2020). Four unique RCTs (N-MOmentum, PREVENT, SAkuraSky, and SAkuraStar) were identified, and data from 29 publications were extracted for analysis. Network scenarios describing the most comparable patient population groups (such as by treatment settings) were evaluated in our analyses. Relative treatment effects were evaluated based on time-to-first relapse and were expressed as hazard ratios (HRs) with 95\% credible intervals (CrIs).

Results: In patients treated with a monoclonal antibody only, eculizumab was associated with a

I. Nakashima

Tohoku Medical and Pharmaceutical University, Sendai, Japan

F. Paul

Charité-Universitätsmedizin Berlin, Berlin, Germany

F. Paul

Max Delbrueck Center for Molecular Medicine, Berlin, Germany

S. J. Pittock ( $\square)$

Mayo Clinic, 200 1st St SW, Rochester, MN 55905, USA

e-mail: pittock.sean@mayo.edu 
lower risk of relapse compared with satralizumab (HR 0.10, 95\% CrI 0.01, 0.65) and inebilizumab (HR 0.11, 95\% CrI 0.02, 0.68). In patients treated with monoclonal antibody with or without background immunosuppressive therapy (IST), patients treated with eculizumab \pm IST were also less likely to relapse than patients treated with satralizumab \pm IST (HR 0.24, 95\% CrI 0.06, 0.98). Conclusion: The NMA results suggest that complement component 5 (C5) inhibition prevents NMOSD relapses more effectively than broader mechanisms of action.

\section{PLAIN LANGUAGE SUMMARY}

Neuromyelitis optica spectrum disorder (NMOSD) is an autoimmune disease characterized by inflammation that damages the brain and spinal cord. Many patients with NMOSD produce antibodies against a protein called aquaporin-4 (AQP4+). In the past two years, three drugs (eculizumab, inebilizumab, and satralizumab) have been approved by the U.S. Food and Drug Administration for the treatment of adults with AQP4+ NMOSD. Comparing the efficacy of these three drugs would help physicians make treatment decisions for their patients. In the absence of clinical trials directly comparing these three drugs, we conducted a Bayesian network meta-analysis in order to allow for simultaneous comparisons of these three drugs and estimate relative treatment effects between any pair of interventions in a connected network. With a Bayesian methodology, it is also possible to estimate the probability of being the best treatment out of all other interventions in a connected network. While all three drugs are safe and shown to prevent relapses in placebo-controlled trials, the results of our analysis suggests that eculizumab was the most efficacious in preventing relapses when compared with inebilizumab or satralizumab. These findings may help to inform physicians and their patients when determining the best treatment option for preventing the occurrence of relapses in adults with AQP4+ NMOSD.

Keywords: Neuromyelitis optica; Aquaporin-4; Network meta-analysis; Eculizumab; Inebilizumab; Satralizumab

\section{Key Summary Points}

\section{Why carry out this study?}

Three drugs (eculizumab, inebilizumab, and satralizumab) are currently approved by the U.S. Food and Drug Administration for the treatment of adults with aquaporin-4 immunoglobulin G-positive neuromyelitis optica spectrum disorder (AQP4+ NMOSD).

In the absence of head-to-head clinical trials, we conducted a systematic literature review to identify data from randomized controlled trials (RCTs) for eculizumab, inebilizumab, and satralizumab and then performed an indirect treatment comparison of their efficacy via a fixed-effects Bayesian network meta-analysis (NMA).

\section{What was learned from this study?}

We found 29 publications from four unique RCTs (N-MOmentum, PREVENT, SAkuraSky, and SAkuraStar); the only efficacy outcome that could be evaluated in the NMA was time-to-first relapse, because it was the only outcome shared across all RCTs in AQP4+ NMOSD.

Hazard ratios suggest that patients on eculizumab monotherapy were $90 \%$ and $89 \%$ less likely to relapse when compared with patients on satralizumab or inebilizumab monotherapy, respectively; in all three treatment-setting scenarios tested (combined mono- and combination therapy, monotherapy, and combination therapy), eculizumab had the greatest likelihood of being the best treatment option for delaying time-to-first relapse in adults with AQP4+ NMOSD.

Our results suggest that complement component 5 (C5) inhibition may prevent NMOSD relapses more effectively than treatments with broader mechanisms of action, such as those targeting the interleukin-6 receptor or the B-cell surface antigen cluster of differentiation 19 (CD19). 


\section{INTRODUCTION}

Neuromyelitis optica spectrum disorder (NMOSD) is a rare, severely disabling, complement-mediated autoimmune neuroinflammatory disease of the central nervous system (CNS), characterized by devastating and unpredictable attacks (relapses) that can cause immediate and irreversible damage [1, 2]. Aquaporin-4 (AQP4) immunoglobulin G-induced activation of the complement cascade is one of the primary drivers of pathogenesis in NMOSD [3-5]. An estimated $80-90 \%$ of NMOSD cases follow a relapsing disease course $[1,6]$. Any relapse can result in the accumulation of neurological disability, including blindness and paralysis, which highlights the immediate need for immunotherapies that effectively prevent NMOSD relapses [7-13].

Eculizumab, the first immunosuppressive monoclonal antibody approved by the U.S. Food and Drug Administration (FDA) to treat adults with AQP4 immunoglobulin (AQP4+) NMOSD, works by inhibiting the complement component 5 (C5) protein in the terminal part of the complement cascade $[7,14]$. In a phase 3 randomized controlled trial (RCT) with a placebo control (PREVENT), eculizumab demonstrated outstanding efficacy in preventing NMOSD relapses in adults with AQP4+ NMOSD; safety outcomes were consistent with its use in other indications (paroxysmal nocturnal hemoglobinuria, atypical hemolytic uremic syndrome, and generalized myasthenia gravis) $[7,14]$. Data suggest that interleukin 6 (IL-6) may also contribute to the inflammation that arises in AQP4+ NMOSD [15]. Satralizumab, a monoclonal antibody which targets the IL-6 receptor (IL-6R), has shown efficacy in a phase 3 RCT (SAkuraSky) in combination with immunosuppressive therapies (ISTs) and as a monotherapy agent (SAkuraStar) $[16,17]$. In addition, inebilizumab, a monoclonal antibody that evokes antibody-dependent cellular cytolysis by binding to the B-cell surface antigen cluster of differentiation 19 (CD19), has been shown to reduce the risk of relapse compared with placebo in a phase $2 / 3$ placebo-controlled RCT (N-MOmentum) among patients with NMOSD [18]. At the time of this analysis, these three therapies had received FDA approval for the treatment of adults with AQP4+ NMOSD. Some of them have also received approval for this indication from additional regulatory agencies, such as the European Medicines Agency and the Pharmaceuticals and Medical Devices Agency of Japan.

With several approved therapies from which to choose, the need for comparative efficacy arises. Healthcare providers consider comparative efficacy to be one of the parameters when selecting a treatment for an individual patient, and patients want to know which drug is most likely to keep them relapse-free. Payers and fundholders want to consider evidence on comparative efficacy to determine coverage, prior authorization, and reimbursement. Headto-head RCTs are considered the most credible sources of evidence to obtain insight into the relative treatment effects of different therapies. However, it is unrealistic to expect head-to-head RCTs to compare all interventions of interest. This is where a network meta-analysis (NMA) can help. With an NMA, the trial-specific treatment effects of several therapies can be compared simultaneously to evaluate their relative efficacy, similar to a head-to-head RCT of all competing treatment options [19]. To our knowledge, no NMA has been performed to date that compares the efficacies of all three FDA-approved treatments in adults with AQP4+ NMOSD. The aim of this study was to evaluate the relative efficacy of all FDA-approved treatments (eculizumab, inebilizumab, and satralizumab) by conducting an NMA based on published data from RCTs in adults with AQP4+ NMOSD.

\section{METHODS}

\section{Identification and Selection of Relevant Trials}

A systematic literature review (SLR) search was conducted on 11 September 2020, focusing on adult patients with AQP4+ NMOSD. Database searches of MEDLINE, EMBASE, and the Cochrane Central Register of Controlled Trials were executed using predefined search strategies in Ovid (Electronic Supplementary Material 
[ESM] Appendix A). After the identification of records, titles and abstracts were screened against the eligibility criteria (see Table 1), and duplicates were removed; full texts of the included records were then reviewed in a second screening round against the same criteria (see Table 1 and ESM Appendix B). Both rounds of screening were performed by two independent reviewers, with any disagreements resolved by a third reviewer. This article is based on previously conducted studies and does not contain any new studies with human participants or animals performed by any of the authors.

\section{Data Extraction and Risk Assessment}

Study quality was assessed by the Cochrane Risk of Bias tool, which evaluated the study randomization process, deviations from intended interventions, missing outcome data, measurement of outcome, and selection of reported results. Each evaluated domain is assigned a bias status of either "low risk," "of some concern," or "high risk" (see ESM Appendix B for additional information on data extraction and risk assessment).

\section{NMA and Outcomes}

A Bayesian NMA was performed to compare the relative treatment effects between eculizumab, inebilizumab, and satralizumab based on data extracted from RCTs identified from our SLR and deemed sufficiently similar. Analyses were performed for clinically relevant subpopulations based on three treatment networks (see Fig. 1):

- Analysis 1: Combined mono- and combination therapy-patients treated with a monoclonal antibody with or without background IST (Fig. 1a).

- Analysis 2: Monotherapy-patients treated with a monoclonal antibody only (no background IST; Fig. 1b).

- Analysis 3: Combination therapy-patients treated with a monoclonal antibody with background IST (Fig. 1c).
Table 1 PICOS screening criteria for the systematic literature review of randomized controlled trials in adults with AQP4+ NMOSD

\begin{tabular}{|c|c|}
\hline Criteria & Inclusion \\
\hline \multirow[t]{2}{*}{ Population } & Target population: \\
\hline & -Adults with AQP4+ NMOSD \\
\hline \multirow[t]{5}{*}{ Interventions } & FDA-approved treatment for \\
\hline & AQP4 + NMOSD: \\
\hline & -Eculizumab \\
\hline & -Satralizumab \\
\hline & -Inebilizumab \\
\hline \multirow[t]{3}{*}{ Comparators } & Placebo or best supportive care \\
\hline & Any intervention of interest \\
\hline & $\begin{array}{l}\text { Any treatment that facilitates an } \\
\text { indirect comparison }\end{array}$ \\
\hline \multirow[t]{14}{*}{ Outcomes $^{a}$} & Efficacy: \\
\hline & -Time-to-first relapse \\
\hline & -Annualized relapse rate \\
\hline & Health-related quality of life: \\
\hline & -Disease-related disability \\
\hline & measured by the Expanded \\
\hline & Disability Status Scale \\
\hline & -Modified Rankin Scale \\
\hline & -Hauser Ambulation Index \\
\hline & -European Quality of Life \\
\hline & 5-Dimension Questionnaire \\
\hline & Index Score \\
\hline & -36-item Short Form Health \\
\hline & Survey \\
\hline Study design & $\mathrm{RCTs}^{\mathrm{a}}$ \\
\hline Language & $\begin{array}{l}\text { Only studies published in English } \\
\text { were included }\end{array}$ \\
\hline Time & No time restriction was used \\
\hline \multicolumn{2}{|l|}{ Data sources } \\
\hline \multirow[t]{3}{*}{ Databases (via Ovid) } & EMBASE \\
\hline & MEDLINE \\
\hline & $\begin{array}{c}\text { Cochrane Central Register of } \\
\text { Controlled Clinical Trials }\end{array}$ \\
\hline
\end{tabular}


Table 1 continued

\begin{tabular}{ll}
\hline Criteria & Inclusion \\
\hline $\begin{array}{l}\text { Conference } \\
\text { proceedings } \\
(2018-2020)\end{array}$ & European Committee for \\
& Treatment and Research in \\
& Multiple Sclerosis \\
& American Academy of Neurology \\
& Annual Meeting \\
Trials in progress & European Academy of Neurology \\
\hline
\end{tabular}

$A Q P 4+$ aquaporin-4 immunoglobulin G-positive, $F D A$ U.S. Food and Drug Administration, NMA network metaanalysis, NMOSD neuromyelitis optica spectrum disorder, PICOS Population, Intervention, Comparison, Outcomes and Study, RCTs randomized controlled trials

${ }^{a} \mathrm{RCT}$ s reporting at least one efficacy outcome were selected for inclusion. All reported efficacy outcomes were extracted from each publication identified. Only data from $\mathrm{AQP} 4+$ patients were included in the NMA

These networks were selected based on our SLR results and an evaluation of the populations deemed most comparable for performing an NMA. All analyses adhered to guidance from the International Society for Pharmacoeconomics and Outcomes Research (ISPOR) network metaanalysis (indirect treatment comparison) Task Force [20].

Results of an NMA are usually expressed in two ways: hazard ratios (HRs) and rank order probabilities. Relative treatment effects are expressed as HRs, which is standard for an NMA [21]. The HR is a comparison of the number of events in a treatment group versus the number of events in a control group, accounting for follow-up time. In NMAs, it is rare to find statistically significant differences. In many cases, this is because the analysis is simply underpowered. As an NMA is more focused on identifying potential treatment effect differences than on hypothesis testing, a Bayesian statistical approach is typically used. This approach simultaneously allows for the calculation of rank order probabilities [19].

The probability that a treatment is best can be described as the proportion of samples where the sampled effect size for one treatment is greater than the sampled effect size of other treatments. The samples are taken from the model's posterior distribution of the relative treatment effect estimates across all treatments compared in a network. Similarly, it is possible to calculate the probability of being the secondbest treatment, the third-best treatment, and so on, up to the probability of being the worst treatment option out of those assessed. These probability statements can be plotted in a "rankogram" and offer an intuitive summary of how treatments rank given their sampled effect size and associated uncertainty. When evaluating HRs and rank order probabilities in tandem, the smaller the HR (i.e., the more effective the treatment), the more likely the treatment will be ranked first when evaluating the probability of being the best treatment option. This information can help to inform clinical decisionmaking based on treatment efficacy $[19,22]$.

In our NMA, the key efficacy outcome of time-to-first relapse-defined as the presence or worsening of neurologic symptoms related to NMOSD_was evaluated using a regression model with a contrast-based normal likelihood for the $\log$ hazard ratio (logHR) and corresponding standard error of each trial (or comparison) in the network. The NMA was performed under the assumption of proportional hazards. Based on the posterior distributions of the relative treatment effect estimates, rank order probabilities were also calculated.

Time-to-first relapse (assessed by an independent review committee) was the primary and only reported outcome shared across all three RCTs in adults with AQP4+ NMOSD. Additional outcomes analyzed in the RCTs included measures such as annualized relapse rate (ARR), Expanded Disability Status Scale (EDSS) score, Modified Rankin Scale (mRS) score, Hauser Ambulation Index (HAI) score, EuroQol 5-Dimension Questionnaire (EQ-5D) index score, and 36-Item Short Form Survey (SF36) score. However, these results were not available for adults with AQP4+ NMOSD in all RCTs. Because of this, an NMA with all secondary endpoints was not feasible and would have introduced bias into the analyses. Therefore, the NMA focused only on the primary 
a

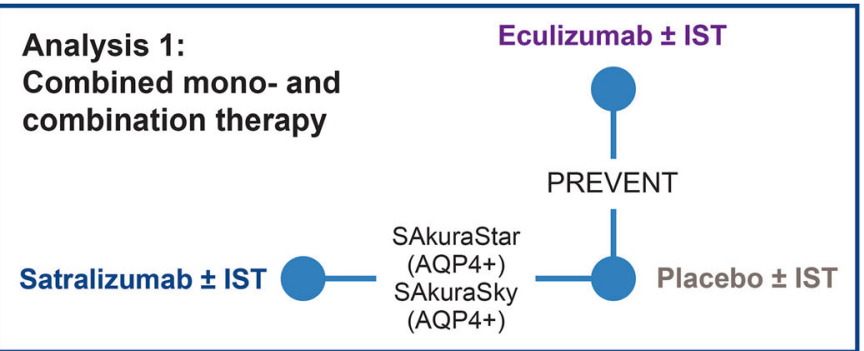

b

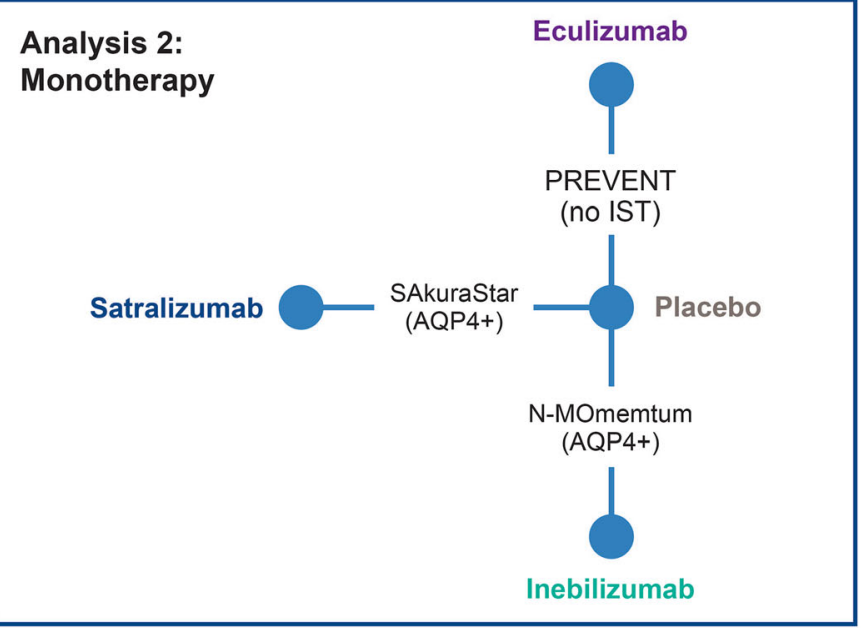

c
Analysis 3:

Combination therapy

Satralizumab + IST

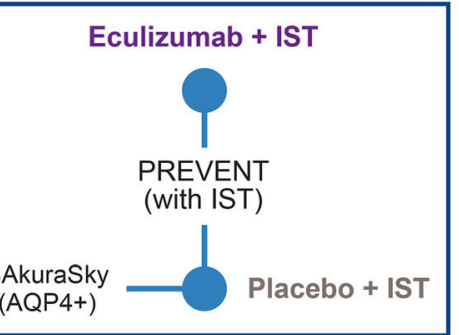

\begin{tabular}{|c|c|c|c|c|c|}
\hline Network & $\begin{array}{l}\text { Eculizumab } \\
\text { (PREVENT) }\end{array}$ & $\begin{array}{l}\text { Satralizumab } \\
\text { (SAkuraStar) }\end{array}$ & $\begin{array}{l}\text { Satralizumab } \\
\text { (SAkuraSky) }\end{array}$ & $\begin{array}{l}\text { Inebilizumab } \\
\text { (N-MOmentum) }\end{array}$ & $\begin{array}{c}\text { Placebo } \\
\text { (pooled from RCTs) }\end{array}$ \\
\hline $\begin{array}{l}\text { Analysis 1: } \\
\text { Combined } \\
\text { mono- and } \\
\text { combination } \\
\text { therapy }\end{array}$ & $N=96$ & \multicolumn{2}{|c|}{$N=68$} & & $N=98$ \\
\hline $\begin{array}{l}\text { Analysis 2: } \\
\text { Monotherapy }\end{array}$ & $N=21$ & $N=41$ & & $N=161$ & $N=122$ \\
\hline $\begin{array}{l}\text { Analysis } 3: \\
\text { Combination } \\
\text { therapy }\end{array}$ & $N=75$ & & $N=27$ & & $N=75$ \\
\hline
\end{tabular}

Fig. 1 Network meta-analysis design: indirect treatment comparison of time-to-first relapse in adults with AQPQ+ NMOSD. In the SAkuraSky and PREVENT studies, background IST, such as azathioprine, mycophenolate mofetil, and glucocorticoids, were allowed, whereas IST was explicitly excluded from the N-MOmentum and SAkuraStar populations. $A Q P Q+$ aquaporin- 4 immunoglobulin G-positive, IST immunosuppressant therapy, NMOSD neuromyelitis spectrum disorder 
efficacy outcome of time-to-first relapse in patients with AQP4+ NMOSD. As such, only data from patients with AQP4+ NMOSD were included in the NMA.

\section{Software}

The parameters of the different models were estimated using a Markov chain Monte Carlo (MCMC) method implemented in the JAGS software package. A first series of iterations from the JAGS sampler was discarded as 'burn-in' and the inferences were based on additional iterations using two chains. All analyses were performed using $\mathrm{R}$ version 3.6.1 and JAGS version 4.3.0.

\section{RESULTS}

\section{SLR and PRISMA Flow Chart}

Four unique studies (N-MOmentum, PREVENT, SAkuraSky, and SAkuraStar) were identified in 29 publications retrieved from the databases during the SLR (search end date: 11 September 2020). A PRISMA (Preferred Reporting Items for Systematic Reviews and Meta-Analyses) flow diagram summarizing this study selection process is shown in Fig. 2. Patient and trial characteristics can be found in ESM Appendices C, $\mathrm{D}$, and $\mathrm{E}$. Seven additional records were identified from conference proceedings of the European Committee for Treatment and Research in Multiple Sclerosis (ECTRIMS), American Academy of Neurology (AAN), and European Academy of Neurology (EAN). In total, 29 publications representing four RCTs met the Population, Intervention, Comparison, Outcomes, and Study (PICOS) screening criteria: N-MOmentum (inebilizumab), PREVENT (eculizumab), SAkuraSky (satralizumab), and SAkuraStar (satralizumab; see Table 1). Of the 29 publications, HR data were pulled from the four primary RCTs, and the remaining 25 were abstracts (see ESM Table S4). In all trials, active treatment with monoclonal antibodies demonstrated significantly favorable efficacy profiles when compared with placebo.

\section{Treatment Effects}

Analysis 1: Combined Mono- and Combination Therapy-Eculizumab \pm IST has the Highest Likelihood of Being the Best Treatment Option for Extending Time-to-First Relapse

The HRs for time-to-first relapse in a mixed population of patients on monotherapy or combination therapy were determined from three trials evaluating eculizumab and satralizumab. The relative treatment effect of satralizumab \pm IST was obtained by pooling the results of the SAkuraStar (monotherapy) and SAkuraSky (combination therapy) trials. The HR results indicate that patients treated with eculizumab \pm IST are $76 \%$ less likely to experience a first relapse compared with satralizumab \pm IST (HR 0.24, 95\% credible interval [CrI] 0.06, 0.98; Fig. 3). When calculating rank order probabilities, eculizumab \pm IST demonstrated the highest probability (98\%) of being the best treatment option for delaying time-to-first relapse in adults with AQP4+ NMOSD (see Table 2).

\section{Analysis 2: Monotherapy-Patients on Eculizumab Monotherapy Were Less Likely to Experience a First Relapse Compared with Satralizumab Monotherapy or Inebilizumab Monotherapy}

Time-to-first relapse was captured by three trials evaluating all three monoclonal antibodies of interest. The monotherapy network was composed of the N-MOmentum, PREVENT (no IST), and SAkuraStar trials. Results showed that patients on eculizumab monotherapy were $90 \%$ less likely to experience a first relapse when compared with satralizumab monotherapy (HR 0.10, 95\% CrI 0.01, 0.65) and 89\% less likely to experience a first relapse when compared with inebilizumab monotherapy (HR 0.11, 95\% CrI 0.02, 0.68; Fig. 3). Of note, patients on eculizumab monotherapy did not experience a relapse during the PREVENT study. Because it is not possible to conduct an NMA with an HR of zero, an HR of 0.025 was imputed using the Firth correction [23].

In addition, eculizumab monotherapy had the highest probability (99\%) of being the best treatment option for improving time-to-first relapse in adults with AQP4+ NMOSD (Table 2). 


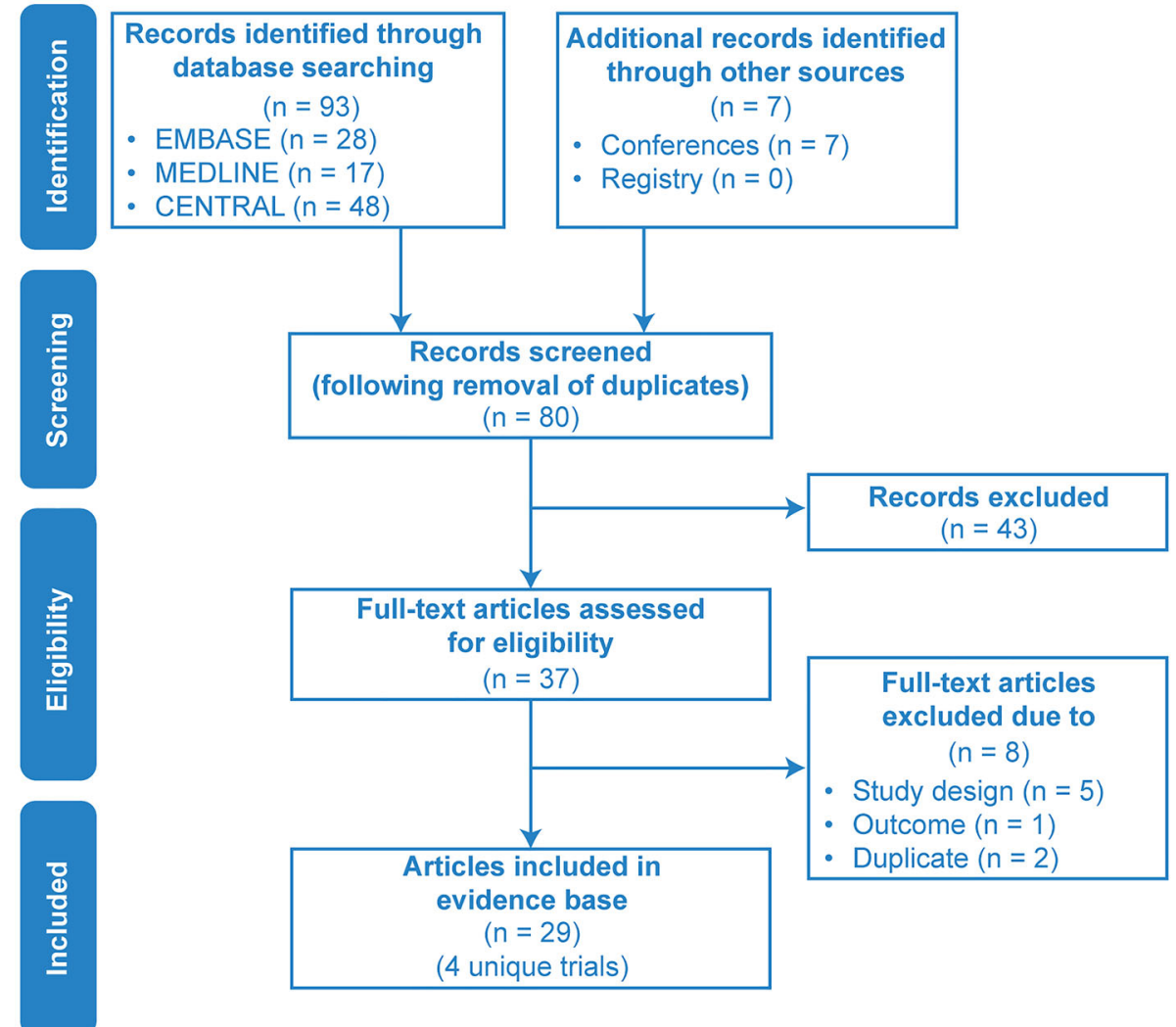

Fig. 2 PRISMA diagram: RCT identification and selection. CENTRAL refers to the Cochrane Central Register of Controlled Trials. PRISMA Preferred Reporting Items for Systematic Reviews and Meta-Analyses, RCT randomized controlled trial

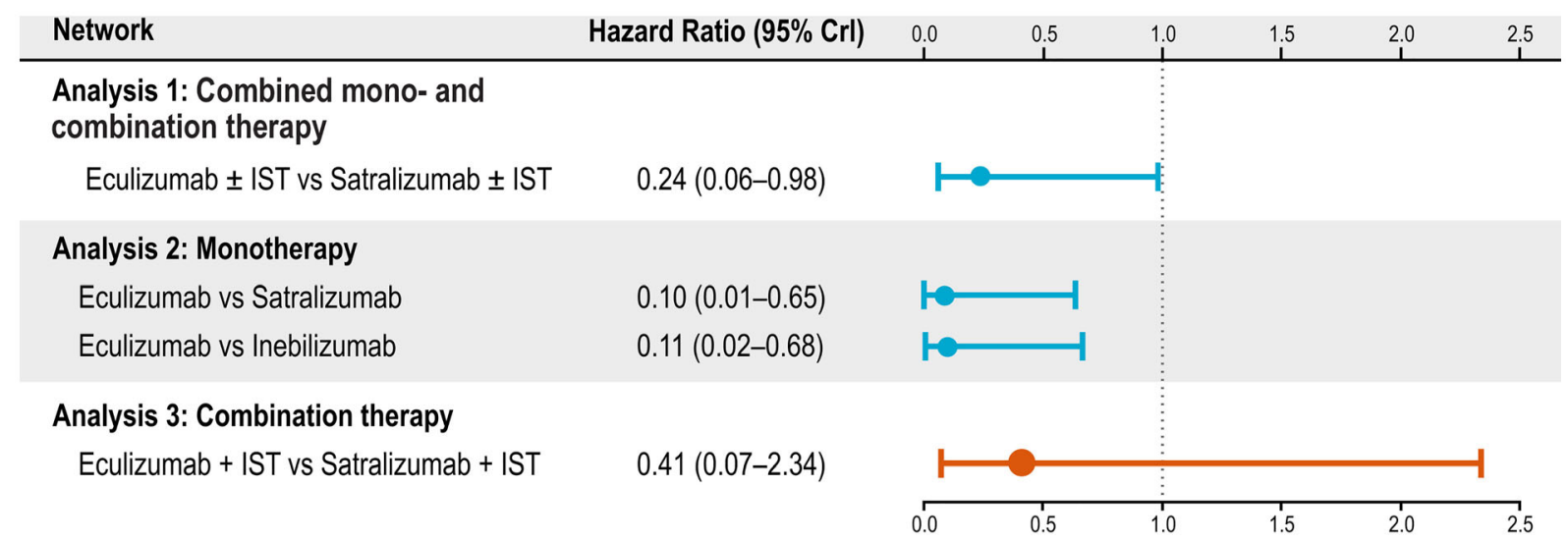

Fig. 3 Hazard ratios for time-to-first relapse in adults with AQP4+ NMOSD. In the SAkuraSky and PREVENT trials, background IST, such as azathioprine, mycophenolate mofetil, and glucocorticoids, were allowed, whereas
IST was explicitly excluded from the N-MOmentum and SAkuraStar populations. $\mathrm{CrI}$ credible interval, IST immunosuppressive therapy 
Table 2 Rank order probabilities of the first rank: likelihood of being the best treatment option for time-to-first relapse in adults with AQP4+ NMOSD

\begin{tabular}{llll}
\hline Treatment & $\begin{array}{l}\text { Analysis 1: combined mono- and } \\
\text { combination therapy }\end{array}$ & $\begin{array}{l}\text { Analysis 2: } \\
\text { monotherapy }\end{array}$ & $\begin{array}{l}\text { Analysis 3: combination } \\
\text { therapy }\end{array}$ \\
\hline Eculizumab & $97.63 \%$ & $98.67 \%$ & $84.47 \%$ \\
Inebilizumab $^{\mathrm{a}}$ & - & $00.74 \%$ & - \\
Satralizumab & $02.37 \%$ & $00.59 \%$ & $15.53 \%$ \\
Placebo & 0 & 0 & 0 \\
\hline
\end{tabular}

AQP4+ aquaporin-4 immunoglobulin G-positive, NMOSD neuromyelitis optica spectrum disorder

${ }^{a}$ Inebilizumab only has monotherapy data and was not included in Analysis 1 or 3

\section{Analysis 3: Combination Therapy- \\ Eculizumab has the Highest Probability \\ of Being the Best Treatment Option for Time- \\ to-First Relapse When Administered in Combination with IST}

The NMA showed that treatment effects for time-to-first relapse between eculizumab + IST and satralizumab + IST were comparable (Fig. 3). Although there was a trend towards a better HR for eculizumab + IST, the differences in treatment effects were too uncertain to determine statistically if one was more favorable than the other (HR 0.41, 95\% CrI 0.07, 2.34). However, rank order probability analyses indicated that eculizumab still had the highest probability $(84 \%)$ of being the best treatment option for delaying time-to-first relapse in adults with AQP4+ NMOSD (Table 2).

Although additional endpoints, such as ARR, disability, heath-related quality of life (HRQoL), and safety outcomes were reported for the RCTs, they could not be evaluated in our NMA without introducing bias. Thus, the raw data for these outcomes were extracted from the publications and are included in ESM Appendix F.

\section{DISCUSSION}

In the combined mono- and combination therapy network, patients treated with eculizumab were less likely to experience a first relapse than patients treated with satralizumab. As a monotherapy, eculizumab was more efficacious than inebilizumab or satralizumab. When combined with IST (combination therapy network), eculizumab may also be more efficacious compared with satralizumab (84\% probability), but the relative treatment effect estimate is too uncertain to claim a significant difference at this time. However, given the post hoc nature of NMAs, it may be beneficial for decision-making to note that our study found significant differences in favor of eculizumab in two out of three treatment comparison scenarios.

Unfortunately, analyses for ARR, disability, and HRQoL were not performed because of a lack of consistent outcome when reported by AQP4+ status in the RCTs $[16,17]$. Safety outcomes were also excluded from the NMA because of a lack of standardized baseline risks and inconsistent reporting by AQP4+ status across trials.

The comparative analysis of these treatments' efficacies is intended to assist the selection of the most appropriate therapy for an individual patient. Although efficacy is an essential parameter when physicians are deciding on a specific treatment, additional factors are considered as part of the decision-making process for a chronic treatment, such as treatment history, patient's preference with regards to the mode and frequency of the drug's administration, established drug's safety profile, and insurance coverage.

\section{Strengths and Limitations}

This is a replicable analysis to estimate relative treatment effects between the primary competing interventions for NMOSD based on a 
commonly accepted methodology using evidence from pivotal RCTs [20]. This analysis estimates what a true head-to-head trial involving all these interventions would likely have shown.

No two trials enroll identical patients. The impact of differences in patient characteristics on absolute results is neutralized by focusing on the relative treatment effect, meaning the incremental, additional benefit is calculated as the difference between the active and the control arm. For an NMA to be deemed credible, RCTs should be as similar as possible in terms of relative treatment effect modifiers. The authors believe this to be the case in this analysis, because our evidence base includes similar RCTs, each comparing a subset of the interventions versus placebo, and the evidence from our SLR can be organized within a single network of studies in which each has at least one intervention group or placebo group corresponding with another trial. As such, the results of our NMA can be used to compare relative treatment effects between interventions of interest [27].

In our analysis, a major characteristic that differed between studies was the presence or absence of a background IST. By performing the analyses stratified by monotherapy and combination therapy applications, we effectively adjusted for this difference. Additionally, these stratified analyses are arguably more beneficial and relevant for clinical decision-making. For the NMA of the mixed combined mono- and combination therapy population, the relative treatment effects of the SAkuraSky and SAkuraStar trials were pooled to get a relative treatment effect representative of this mixed population.

This NMA was conducted using data from a limited number of studies (attempting to match one study per drug) and, therefore, it did not allow for any between-trial adjustment of study characteristics, such as by means of a meta-regression analysis. Another limitation of the current analysis is that no long-term follow-up data of the identified RCTs (i.e., extension periods) were incorporated. The lack of a placebo arm in the long-term extension arm precludes us from calculating the relative treatment effect because it would require significant assumptions about the data.

Additionally, the attack or relapse definition was not completely uniform across the studies; for example, N-MOmentum used magnetic resonance imaging whereas others did not [28]. However, all three studies had independent panels that adjudicated relapses. The entry criteria also varied from study to study, as well as the IST restrictions (i.e., number of drugs that can be used in combination). Lastly, our analyses were limited by the shorter study period of the N-MOmentum trial relative to the other trials. N-MOmentum had a duration of only 197 days, compared with 144 weeks for the other trials. Differences in study duration will bias an NMA if HRs are not constant over time. In N-MOmentum, tests for the proportionality of hazards were completed that indicated the effect of treatments may be reasonably assumed to be constant. Therefore, the shorter duration period of this trial is likely not a strong source of bias to our NMA.

Data from an RCT conducted to explore the efficacy of rituximab versus placebo were recently published (RIN-1) [24], but these data were not included in this analysis. All studies included in this analysis required patients to have a history of relapses during the 12 to 24 months before study start. The RIN-1 study did not share this requirement.

\section{CONCLUSION}

In the absence of head-to-head trials, this NMA provides important evidence on the relative efficacy of eculizumab compared with other approved treatments for NMOSD. Based on currently available RCT evidence, monotherapy and combined mono- and combination therapy with eculizumab are more efficacious at preventing relapses than inebilizumab or satralizumab in the treatment of adults with AQP4+ NMOSD. These findings suggest that C5 inhibition prevents relapses more effectively than broader mechanisms of action involving IL-6R or CD19 inhibition among adults with AQP4+ NMOSD. 


\section{ACKNOWLEDGEMENTS}

Funding. Financial support for the research, authorship, and/or publication of this article: research funding and publication fees, including the journal's Rapid Service Fee, were provided by Alexion, AstraZeneca Rare Disease.

Medical Writing. Medical writing support was provided by Danielle E. Dalechek, MS, and Steven F. Merkel, PhD, of Oxford PharmaGenesis, Inc., Newtown, PA, and was funded by Alexion Pharmaceuticals, Inc.

Authorship. All named authors meet the International Committee of Medical Journal Editors (ICMJE) criteria for authorship for this article, take responsibility for the integrity of the work as a whole, and have given their approval for this version to be published.

Authors' Contributions. Conception and design (all authors); acquisition of data (IZ, AK, $\mathrm{MR}$ ); analysis or interpretation of data (all authors); preparation of manuscript (all authors); critical review of manuscript (all authors). The study sponsor reviewed the analysis plan and study manuscript. All aspects of the SLR (e.g., searches, screening, extraction), data management, processing, and analyses were conducted by PRECISIONheor. All final analytic decisions were made by study investigators. All authors have read and approved the final version of the manuscript.

Disclosures. Imran Tanvir was an employee and stockholder of Alexion Pharmaceuticals during the development of this manuscript. Adrian Kielhorn is an employee and stockholder of Alexion Pharmaceuticals. Minying Royston was an employee and stockholder of Alexion Pharmaceuticals during the development of this manuscript. Michael Levy is an employee of Massachusetts General Hospital and has received consulting fees and grants from Alexion Pharmaceuticals, Viela Bio, and Genentech relevant to the manufacturers of the three drugs mentioned in this abstract. Ichiro Nakashima is an employee of Tohoku Medical and
Pharmaceutical University and has received speech honoraria and consulting fees from Alexion Pharmaceuticals and Chugai Pharmaceutical. Kazuo Fujihara received honoraria for lectures and presentations from Alexion Pharmaceuticals, Inc., Roche/Chugai, and Viela Bio, and served on their advisory boards. Sean J Pittock and his institution have received honoraria and travel expenses for attending NMOSD advisory board meetings (MedImmune, Alexion Pharmaceuticals, Inc.); consulting fees (Alexion Pharmaceuticals, Inc., Euroimmun, Grifols, MedImmune); grant/research support (Alexion Pharmaceuticals, Inc., the Autoimmune Encephalitis Alliance, Euroimmun, Grifols, MedImmune); and other fees from the National Institutes of Health and the Guthy-Jackson Charitable Foundation. Dean M Wingerchuk is an employee of the Mayo Clinic and participated on a data safety monitoring or advisory board for Roche, Viela Bio, Genentech, Biogen, Reistone, TG Therapeutics, Celgene, and Novartis, with fees paid directly to himself; and for Alexion Pharmaceuticals, with fees paid to his institution. He received grants for clinical trials through Alexion Pharmaceuticals and TerumoBCT paid directly to his institution and was personally paid consulting fees by Mitsubishi Tanabe. Ina Zhang is an employee of PRECISIONheor, who received funding from Alexion Pharmaceuticals to support this work. Friedemann Paul is an employee of CharitéUniversitätsmedizin Berlin and has received honoraria for lectures and research support from Alexion Pharmaceuticals. He has received grants for research support/recipient charité from German Ministry for Education and Research (BMBF), Deutsche Forschungsgemeinschaft (DFG), Einstein Foundation, the GuthyJackson Charitable Foundation, EU FP7 Framework Program, Biogen, Genzyme, Merck Serono, Novartis, Bayer, Roche, Parexel, and Almirall. He has received personal payment and travel expenses for lectures, presentations, and speakers' bureaus from the Guthy-Jackson Foundation, Bayer, Biogen, Merck Serono, Sanofi Genzyme, Novartis, Viela Bio, Roche, UCB, Mitsubishi Tanabe, and Celgene. He has participated on an advisory board for Celgene, Roche, UCB, and Merck. He has offered unpaid 
support as an Academic Editor for PLos One and as Associate Editor for Neurology: Neuroimmunology \& Neuroinflammation.

Compliance with Ethics Guidelines. This article is based on previously conducted studies and does not contain any new studies with human participants or animals performed by any of the authors.

Data Availability. Data sharing is not applicable to this article as no datasets were generated or analyzed during the current study. All data for this manuscript were publicly available and can be accessed using the criteria described in the Methods and Supplementary Material.

Open Access. This article is licensed under a Creative Commons Attribution-NonCommercial 4.0 International License, which permits any non-commercial use, sharing, adaptation, distribution and reproduction in any medium or format, as long as you give appropriate credit to the original author(s) and the source, provide a link to the Creative Commons licence, and indicate if changes were made. The images or other third party material in this article are included in the article's Creative Commons licence, unless indicated otherwise in a credit line to the material. If material is not included in the article's Creative Commons licence and your intended use is not permitted by statutory regulation or exceeds the permitted use, you will need to obtain permission directly from the copyright holder. To view a copy of this licence, visit http://creativecommons.org/licenses/by$\mathrm{nc} / 4.0 /$.

\section{REFERENCES}

1. Jarius S, Ruprecht K, Wildemann B, et al. Contrasting disease patterns in seropositive and seronegative neuromyelitis optica: a multicentre study of 175 patients. J Neuroinflamm. 2012;9:14.

2. Hyun JW, Kim Y, Kim SY, Lee MY, Kim SH, Kim HJ. Investigating the presence of interattack astrocyte damage in neuromyelitis optica spectrum disorder: longitudinal analysis of serum glial fibrillary acidic protein. Neurol Neuroimmunol Neuroinflamm. 2021;8(3):e965.
3. Huda S, Whittam D, Bhojak M, et al. Neuromyelitis optica spectrum disorders. Clin Med. 2019;19(2): 169-76.

4. Duan T, Smith AJ, Verkman AS. Complement-dependent bystander injury to neurons in AQP4-IgG seropositive neuromyelitis optica. J Neuroinflamm. 2018;15(1):294.

5. Jarius S, Paul F, Weinshenker BG, Levy M, Kim HJ, Wildemann B. Neuromyelitis optica. Nat Rev Dis Primers. 2020;6(1):85.

6. Akaishi T, Misu T, Takahashi T, et al. Progression pattern of neurological disability with respect to clinical attacks in anti-MOG antibody-associated disorders. J Neuroimmunol. 2021;351:577467.

7. Pittock SJ, Berthele A, Fujihara K, Kim HJ, Levy M, Palace J, et al. Eculizumab in aquaporin-4-positive neuromyelitis optica spectrum disorder. N Engl J Med. 2019;381(7):614-25.

8. Hamid SHM, Whittam D, Mutch K, et al. What proportion of AQP4-IgG-negative NMO spectrum disorder patients are MOG-IgG positive? A cross sectional study of 132 patients. J Neurol. 2017;264(10):2088-94.

9. Wingerchuk DM. Diagnosis and treatment of neuromyelitis optica. Neurologist. 2007;13(1):2.

10. Hinson SR, Romero MF, Popescu BF, et al. Molecular outcomes of neuromyelitis optica (NMO)-IgG binding to aquaporin- 4 in astrocytes. Proc Natl Acad Sci USA. 2012;109(4):1245-50.

11. Morrow MJ, Wingerchuk D. Neuromyelitis optica. J Neuro-ophthalmol. 2012;32(2):154.

12. Levy M, Fujihara K, Palace J. New therapies for neuromyelitis optica spectrum disorder. Lancet Neurol. 2021;20(1):60-7.

13. Kim SH, Jang H, Park NY, et al. Discontinuation of immunosuppressive therapy in patients with neuromyelitis optica spectrum disorder with aquaporin-4 antibodies. Neurol Neuroimmunol Neuroinflamm. 2021;8(2):e947.

14. European Medicines Agency. Soliris: product information. 2021. https://www.ema.europa.eu/en/medi cines/human/EPAR/soliris. Accessed 3 June 2021.

15. Fujihara K, Bennett JL, de Seze J, et al. Interleukin-6 in neuromyelitis optica spectrum disorder pathophysiology. Neurol Neuroimmunol Neuroinflamm. 2020;7(5):e841-e.

16. Yamamura T, Kleiter I, Fujihara $\mathrm{K}$, et al. Trial of satralizumab in neuromyelitis optica spectrum disorder. N Engl J Med. 2019;381(22):2114-24. 
17. Traboulsee A, Greenberg BM, Bennett JL, et al. Safety and efficacy of satralizumab monotherapy in neuromyelitis optica spectrum disorder: a randomised, double-blind, multicentre, placebo-controlled phase 3 trial. Lancet Neurol. 2020;19(5): 402-12.

18. Cree BAC, Bennett JL, Kim HJ, et al. Inebilizumab for the treatment of neuromyelitis optica spectrum disorder (N-MOmentum): a double-blind, randomised placebo-controlled phase $2 / 3$ trial. Lancet. 2019;394(10206):1352-63.

19. Tonin FS, Rotta I, Mendes AM, Pontarolo R. Network meta-analysis: a technique to gather evidence from direct and indirect comparisons. Pharm Pract (Granada). 2017;15(1):943.

20. Jansen JP, Trikalinos T, Cappelleri JC, et al. Indirect treatment comparison/network meta-analysis study questionnaire to assess relevance and credibility to inform health care decision making: an ISPORAMCP-NPC Good Practice Task Force report. Value Health. 2014;17(2):157-73.

21. Jansen JP, Naci H. Is network meta-analysis as valid as standard pairwise meta-analysis? It all depends on the distribution of effect modifiers. BMC Med. 2013;11(1):159.

22. Salanti G, Ades AE, Ioannidis JPA. Graphical methods and numerical summaries for presenting results from multiple-treatment meta-analysis: an overview and tutorial. J Clin Epidemiol. 2011;64(2): 163-71.

23. Firth D. Bias reduction of maximum likelihood estimates. Biometrika. 1993;80(1):27-38.

24. Tahara M, Oeda T, Okada K, et al. Safety and efficacy of rituximab in neuromyelitis optica spectrum disorders (RIN-1 study): a multicentre, randomised, double-blind, placebo-controlled trial. Lancet Neurol. 2020;19(4):298-306.

25. Trebst C, Jarius S, Berthele A, et al. Update on the diagnosis and treatment of neuromyelitis optica: recommendations of the Neuromyelitis Optica Study Group (NEMOS). J Neurol. 2014;261(1):1-16.

26. Pittock SJ, Fujihara K, Palace J, et al. Eculizumab monotherapy for NMOSD: Data from PREVENT and its open-label extension. Mult Scler. 2021. https:// doi.org/10.1177/13524585211038291.

27. Dias S, Welton NJ, Sutton AJ, Caldwell DM, Lu G, Ades AE. Evidence synthesis for decision making 4: inconsistency in networks of evidence based on randomized controlled trials. Med Decis Making. 2013;33(5):641-56.

28. Cree BA, Bennett JL, Sheehan M, et al. Placebocontrolled study in neuromyelitis optica-Ethical and design considerations. Mult Scler. 2016;22(7): 862-72. 